\title{
STABILITY OF LINEAR FUNCTIONAL EQUATIONS IN BANACH MODULES
}

\author{
CHUN-GIL PARK
}

\begin{abstract}
We prove the Hyers-Ulam-Rassias stability of the linear functional equation in Banach modules over a unital Banach algebra.
\end{abstract}

\section{Introduction}

In 1940, S. M. Ulam [12] raised the following question: Under what conditions does there exist an additive mapping near an approximately additive mapping?

Let $E_{1}$ and $E_{2}$ be Banach spaces. Hyers [5] showed that if $\epsilon>0$ and $f: E_{1} \rightarrow E_{2}$ such that

$$
\|f(x+y)-f(x)-f(y)\| \leq \epsilon
$$

for all $x, y \in E_{1}$, then there exists a unique additive mapping $T: E_{1} \rightarrow E_{2}$ such that

$$
\|f(x)-T(x)\| \leq \epsilon
$$

for all $x \in E_{1}$.

Consider $f: E_{1} \rightarrow E_{2}$ to be a mapping such that $f(t x)$ is continuous in $t \in \mathbb{R}$ for each fixed $x \in E_{1}$. Assume that there exist constants $\epsilon \geq 0$ and $p \in[0,1)$ such that

$$
\|f(x+y)-f(x)-f(y)\| \leq \epsilon\left(\|x\|^{p}+\|y\|^{p}\right)
$$

for all $x, y \in E_{1}$. Th. M. Rassias [9] showed that there exists a unique $\mathbb{R}$-linear mapping $T: E_{1} \rightarrow E_{2}$ such that

$$
\|f(x)-T(x)\| \leq \frac{2 \epsilon}{2-2^{p}}\|x\|^{p}
$$

for all $x \in E_{1}$. Gajda [3] generalized the Rassias' result.

Throughout this paper, let $B$ be a unital Banach algebra with norm $|\cdot|, B_{1}=\{a \in$ $B|| a \mid=1\}, \mathbb{R}^{+}$the set of nonnegative real numbers, and let ${ }_{B} M_{1}$ and ${ }_{B} M_{2}$ be left Banach $B$-modules with norms $\|\cdot\|$ and $\|\cdot\|$, respectively. Let $d$ and $s$ be positive integers.

In this paper, we are going to prove the Hyers-Ulam-Rassias stability of the linear functional equation in Banach modules over a unital Banach algebra.

Received March 5, 2002.

2000 Mathematics Subject Classification. Primary 39B72, 39B52, Secondary 46Bxx.

Key words and phrases. Stability, B-linear, Banach algebra.

This work was supported by grant No. R05-2003-000-10006-0 from the Basic Research Program of the Korea Science \& Engineering Foundation. 


\section{Stability of the Linear Functional Equation in Banach Modules}

In this section, we prove the Hyers-Ulam-Rassias stability of the linear functional equation in Banach modules over a unital Banach algebra.

Theorem 2.1. Let $f:{ }_{B} M_{1} \rightarrow{ }_{B} M_{2}$ be a mapping for which there exists a function $\varphi:{ }_{B} M_{1} \times{ }_{B} M_{1} \rightarrow[0, \infty)$ such that

$$
\begin{gathered}
\widetilde{\varphi}(x, y)=\sum_{k=0}^{\infty} 2^{-k} \varphi\left(2^{k} x, 2^{k} y\right)<\infty, \\
\left\|f\left(a^{d} x+a^{d} y\right)-a^{s} f(x)-a^{s} f(y)\right\| \leq \varphi(x, y), \\
\| f(t x+t y)-t f(x)-t f(y) \leq \varphi(x, y)
\end{gathered}
$$

for all $a \in B_{1}$, all $t \in \mathbb{R}^{+}$, and all $x, y \in{ }_{B} M_{1}$. Then there exists a unique $\mathbb{R}$-linear mapping $T:{ }_{B} M_{1} \rightarrow{ }_{B} M_{2}$ such that

$$
\begin{aligned}
T\left(a^{d} x\right) & =a^{s} T(x), \\
\|f(x)-T(x)\| & \leq \frac{1}{2} \widetilde{\varphi}(x, x)
\end{aligned}
$$

for all $a \in B_{1}$ and all $x \in{ }_{B} M_{1}$.

Proof. Let $a=1 \in B_{1}$. By the Găvruta result [4], it follows from the second inequality of the statement that there exists a unique additive mapping $T:{ }_{B} M_{1} \rightarrow{ }_{B} M_{2}$ satisfying (ii). The mapping $T:{ }_{B} M_{1} \rightarrow{ }_{B} M_{2}$ was given by $T(x)=\lim _{n \rightarrow \infty} \frac{f\left(2^{n} x\right)}{2^{n}}$ for all $x \in{ }_{B} M_{1}$.

By the assumption, for each $a \in B_{1}$,

$$
\left\|f\left(2^{n} a^{d} x\right)-2 a^{s} f\left(2^{n-1} x\right)\right\| \leq \varphi\left(2^{n-1} x, 2^{n-1} x\right)
$$

for all $x \in{ }_{B} M_{1}$. Using the fact that for each $a \in B$ and each $z \in{ }_{B} M_{2}\|a z\| \leq K|a| \cdot\|z\|$ for some $K>0$, one can show that

$$
\left\|a^{s} f\left(2^{n} x\right)-2 a^{s} f\left(2^{n-1} x\right)\right\| \leq K\left|a^{s}\right| \cdot\left\|f\left(2^{n} x\right)-2 f\left(2^{n-1} x\right)\right\| \leq K \varphi\left(2^{n-1} x, 2^{n-1} x\right)
$$

for all $a \in B_{1}$ and all $x \in{ }_{B} M_{1}$. So

$$
\begin{aligned}
\left\|f\left(2^{n} a^{d} x\right)-a^{s} f\left(2^{n} x\right)\right\| & \leq\left\|f\left(2^{n} a^{d} x\right)-2 a^{s} f\left(2^{n-1} x\right)\right\|+\left\|2 a^{s} f\left(2^{n-1} x\right)-a^{s} f\left(2^{n} x\right)\right\| \\
& \leq \varphi\left(2^{n-1} x, 2^{n-1} x\right)+K \varphi\left(2^{n-1} x, 2^{n-1} x\right)
\end{aligned}
$$

for all $a \in B_{1}$ and all $x \in{ }_{B} M_{1}$. Thus $2^{-n}\left\|f\left(2^{n} a^{d} x\right)-a^{s} f\left(2^{n} x\right)\right\| \rightarrow 0$ as $n \rightarrow \infty$ for all $a \in B_{1}$ and all $x \in{ }_{B} M_{1}$. Hence

$$
T\left(a^{d} x\right)=\lim _{n \rightarrow \infty} \frac{f\left(2^{n} a^{d} x\right)}{2^{n}}=\lim _{n \rightarrow \infty} \frac{a^{s} f\left(2^{n} x\right)}{2^{n}}=a^{s} T(x)
$$

for each $a \in B_{1}$. 
Similarly, one can obtain that

$$
T(t x)=\lim _{n \rightarrow \infty} \frac{f\left(2^{n} t x\right)}{2^{n}}=\lim _{n \rightarrow \infty} \frac{t f\left(2^{n} x\right)}{2^{n}}=t T(x)
$$

for each $t \in \mathbb{R}^{+}$. Since $t=|t| \cdot \frac{t}{|t|}$ for each $t \in \mathbb{R}(t \neq 0)$,

$$
\begin{aligned}
T\left(t_{1} x+t_{2} y\right) & =T\left(t_{1} x\right)+T\left(t_{2} y\right)=T\left(\left|t_{1}\right| \frac{t_{1}}{\left|t_{2}\right|} x\right)+T\left(\left|t_{2}\right| \frac{t_{2}}{\left|t_{2}\right|} y\right) \\
& =\left|t_{1}\right| T\left(\frac{t_{1}}{\left|t_{1}\right|} x\right)+\left|t_{2}\right| T\left(\frac{t_{2}}{\left|t_{2}\right|} y\right)=\left|t_{1}\right| \frac{t_{1}}{\left|t_{1}\right|} T(x)+\left|t_{2}\right| \frac{t_{2}}{\left|t_{2}\right|} T(y) \\
& =t_{1} T(x)+t_{2} T(y)
\end{aligned}
$$

for all $t_{1}, t_{2} \in \mathbb{R}^{+}\left(t_{1}, t_{2} \neq 0\right)$ and all $x, y \in{ }_{B} M_{1}$. And $T(x)=T(x-y+y)=$ $T(x-y)+T(y)$ for all $x \in{ }_{B} M_{1}$. Hence $T(x-y)=T(x)-T(y)$ for all $x \in{ }_{B} M_{1}$. So the unique additive mapping $T:{ }_{B} M_{1} \rightarrow{ }_{B} M_{2}$ is an $\mathbb{R}$-linear mapping satisfying the conditions given in the statement.

Let $d=s=1$ in Theorem 2.1. Since $T(a x)=a T(x)$ for all $a \in B_{1}$ and all $x \in{ }_{B} M_{1}$,

$$
\begin{aligned}
T(a x+b y) & =T(a x)+T(b y)=T\left(|a| \frac{a}{|a|} x\right)+T\left(|b| \frac{b}{|b|} y\right) \\
& =|a| T\left(\frac{a}{|a|} x\right)+|b| T\left(\frac{b}{|b|} y\right)=|a| \frac{a}{|a|} T(x)+|b| \frac{b}{|b|} T(y) \\
& =a T(x)+b T(y)
\end{aligned}
$$

for all $a, b \in B(a, b \neq 0)$ and all $x, y \in{ }_{B} M_{1}$. And $T(0 x)=0 T(x)$ for all $x \in{ }_{B} M_{1}$. So the $\mathbb{R}$-linear mapping $T:{ }_{B} M_{1} \rightarrow{ }_{B} M_{2}$ is $B$-linear.

Remark 2.1. If the second inequality in the statement of Theorem 2.1 is replaced by

$$
\left\|f\left(a^{d} x+y\right)-a^{s} f(x)-f(y)\right\| \leq \varphi(x, y)
$$

then

$$
\begin{aligned}
& \left\|f\left(a^{d} x+a^{d} y\right)-a^{s} f(x)-f\left(a^{d} y\right)\right\| \leq \varphi\left(x, a^{d} y\right), \\
& \left\|f\left(a^{d} x+a^{d} y\right)-f\left(a^{d} x\right)-a^{s} f(y)\right\| \leq \varphi\left(y, a^{d} x\right), \\
& \left\|f\left(a^{d} x+a^{d} y\right)-f\left(a^{d} x\right)-f\left(a^{d} y\right)\right\| \leq \varphi\left(a^{d} x, a^{d} y\right) .
\end{aligned}
$$

So

$$
\left\|f\left(a^{d} x+a^{d} y\right)-a^{s} f(x)-a^{s} f(y)\right\| \leq \varphi\left(x, a^{d} y\right)+\varphi\left(y, a^{d} x\right)+\varphi\left(a^{d} x, a^{d} y\right),
$$

hence the result does also hold.

Corollary 2.2. Let $f:{ }_{B} M_{1} \rightarrow{ }_{B} M_{2}$ be a mapping for which there exist constants $\epsilon \geq 0$ and $p \in[0,1)$ such that

$$
\begin{array}{r}
\left\|f\left(a^{d} x+a^{d} y\right)-a^{s} f(x)-a^{s} f(y)\right\| \leq \epsilon\left(\|x\|^{p}+\|y\|^{p}\right), \\
\|f(t x+t y)-t f(x)-t f(y)\| \leq \epsilon\left(\|x\|^{p}+\|y\|^{p}\right)
\end{array}
$$


for all $a \in B_{1}$, all $t \in \mathbb{R}^{+}$, and all $x, y \in{ }_{B} M_{1}$. Then there exists a unique $\mathbb{R}$-linear mapping $T:{ }_{B} M_{1} \rightarrow{ }_{B} M_{2}$ such that

$$
\begin{aligned}
T\left(a^{d} x\right) & =a^{s} T(x), \\
\|f(x)-T(x)\| & \leq \frac{2 \epsilon}{2-2^{p}}\|x\|^{p}
\end{aligned}
$$

for all $a \in B_{1}$ and all $x \in{ }_{B} M_{1}$.

Proof. Define $\varphi(x, y)=\epsilon\left(\|x\|^{p}+\|y\|^{p}\right)$, and apply Theorem 2.1.

Corollary 2.3. Let $E_{1}$ and $E_{2}$ be complex Banach spaces and $f: E_{1} \rightarrow E_{2}$ a mapping for which there exists a function $\varphi: E_{1} \times E_{1} \rightarrow[0, \infty)$ such that

$$
\begin{gathered}
\widetilde{\varphi}(x, y)=\sum_{k=0}^{\infty} 2^{-k} \varphi\left(2^{k} x, 2^{k} y\right)<\infty, \\
\left\|f\left(\mu^{d} x+\mu^{d} y\right)-\mu^{s} f(x)-\mu^{s} f(y)\right\| \leq \varphi(x, y), \\
\|f(\lambda x+\lambda y)-\lambda f(x)-\lambda f(y)\| \leq \varphi(x, y)
\end{gathered}
$$

for all $\mu \in \mathbb{C}_{1}=\mathbb{T}^{1}$, all $\lambda \in \mathbb{R}^{+}$, and all $x, y \in E_{1}$. Then there exists a unique $\mathbb{R}$-linear mapping $T: E_{1} \rightarrow E_{2}$ such that

$$
\begin{aligned}
T\left(\mu^{d} x\right) & =\mu^{s} T(x), \\
\|f(x)-T(x)\| & \leq \frac{1}{2} \widetilde{\varphi}(x, x)
\end{aligned}
$$

for all $\mu \in \mathbb{T}^{1}$ and all $x \in E_{1}$.

Proof. Since $\mathbb{C}$ is a unital Banach algebra, the Banach spaces $E_{1}$ and $E_{2}$ are considered as Banach modules over $\mathbb{C}$. By Theorem 2.1, there exists a unique $\mathbb{R}$-linear mapping $T: E_{1} \rightarrow E_{2}$ satisfying the conditions given in the statement.

Now we prove the Hyers-Ulam-Rassias stability of another linear functional equation in Banach modules over a unital Banach algebra.

Theorem 2.4. Let $f:{ }_{B} M_{1} \rightarrow{ }_{B} M_{2}$ be a mapping for which there exists a function $\varphi:{ }_{B} M_{1} \times{ }_{B} M_{1} \rightarrow[0, \infty)$ satisfying $(i)$ such that

$$
\begin{array}{r}
\left\|a^{s} f(x+y)-f\left(a^{d} x\right)-f\left(a^{d} y\right)\right\| \leq \varphi(x, y), \\
\|t f(x+y)-f(t x)-f(t x)\| \leq \varphi(x, y)
\end{array}
$$

for all $a \in B_{1}$, all $t \in \mathbb{R}^{+}$, and all $x, y \in{ }_{B} M_{1}$. Then there exists a unique $\mathbb{R}$-linear mapping $T:{ }_{B} M_{1} \rightarrow{ }_{B} M_{2}$ satisfying (ii) such that

$$
a^{s} T(x)=T\left(a^{d} x\right)
$$

for all $a \in B_{1}$ and all $x \in{ }_{B} M_{1}$. 
Proof. By the same reasoning as the proof of Theorem 2.1, there exists a unique additive mapping $T:{ }_{B} M_{1} \rightarrow{ }_{B} M_{2}$ satisfying (ii).

By the assumption, for each $a \in B_{1}$,

$$
\left\|a^{s} f\left(2^{n} x\right)-2 f\left(2^{n-1} a^{d} x\right)\right\| \leq \varphi\left(2^{n-1} x, 2^{n-1} x\right)
$$

for all $x \in{ }_{B} M_{1}$. So

$$
\begin{aligned}
\left\|a^{s} f\left(2^{n} x\right)-f\left(2^{n} a^{d} x\right)\right\| & \leq\left\|a^{s} f\left(2^{n} x\right)-2 f\left(2^{n-1} a^{d} x\right)\right\|+\left\|2 f\left(2^{n-1} a^{d} x\right)-f\left(2^{n} a^{d} x\right)\right\| \\
& \leq \varphi\left(2^{n-1} x, 2^{n-1} x\right)+\varphi\left(2^{n-1} a^{d} x, 2^{n-1} a^{d} x\right)
\end{aligned}
$$

for all $a \in B_{1}$ and all $x \in{ }_{B} M_{1}$. So $2^{-n}\left\|a^{s} f\left(2^{n} x\right)-f\left(2^{n} a^{d} x\right)\right\| \rightarrow 0$ as $n \rightarrow \infty$ for all $a \in B_{1}$ and all $x \in{ }_{B} M_{1}$. Hence

$$
a^{s} T(x)=\lim _{n \rightarrow \infty} \frac{a^{s} f\left(2^{n} x\right)}{2^{n}}=\lim _{n \rightarrow \infty} \frac{f\left(2^{n} a^{d} x\right)}{2^{n}}=T\left(a^{d} x\right)
$$

for all $a \in B_{1}$.

Similarly, one can obtain that

$$
t T(x)=\lim _{n \rightarrow \infty} \frac{t f\left(2^{n} x\right)}{2^{n}}=\lim _{n \rightarrow \infty} \frac{f\left(2^{n} t x\right)}{2^{n}}=T(t x)
$$

for each $t \in \mathbb{R}^{+}$.

The rest of the proof is the same as the proof of Theorem 2.1. So the unique additive mapping $T:{ }_{B} M_{1} \rightarrow{ }_{B} M_{2}$ is an $\mathbb{R}$-linear mapping satisfying (ii) such that

$$
a^{s} T(x)=T\left(a^{d} x\right)
$$

for all $a \in B_{1}$ and all $x \in{ }_{B} M_{1}$, as desired.

Theorem 2.5. Let $f:{ }_{B} M_{1} \rightarrow{ }_{B} M_{2}$ be a mapping for which there exists a function $\varphi:{ }_{B} M_{1} \times{ }_{B} M_{1} \rightarrow[0, \infty)$ satisfying (i) such that

$$
\begin{aligned}
\|f(t x+t y)-t f(x)-t f(y)\| & \leq \varphi(x, y), \\
\left\|f\left(a^{d} x\right)-a^{s} f(x)\right\| & \leq \varphi(x, x)
\end{aligned}
$$

for all $a \in B_{1}$, all $t \in \mathbb{R}^{+}$, and all $x, y \in{ }_{B} M_{1}$. Then there exists a unique $\mathbb{R}$-linear mapping $T:{ }_{B} M_{1} \rightarrow{ }_{B} M_{2}$ satisfying (ii) such that

$$
T\left(a^{d} x\right)=a^{s} T(x)
$$

for all $a \in B_{1}$ and all $x \in{ }_{B} M_{1}$.

Proof. By the same reasoning as the proof of Theorem 2.1, there exists a unique additive mapping $T:{ }_{B} M_{1} \rightarrow{ }_{B} M_{2}$ satisfying (ii). Combining the definition of the mapping $T$ and the second inequality given in the statement yields that

$$
T\left(a^{d} x\right)=\lim _{n \rightarrow \infty} \frac{f\left(2^{n} a^{d} x\right)}{2^{n}}=\lim _{n \rightarrow \infty} \frac{a^{s} f\left(2^{n} x\right)}{2^{n}}=a^{s} T(x)
$$


for all $a \in B_{1}$ and all $x \in{ }_{B} M_{1}$.

The rest of the proof is the same as the proof of Theorem 2.1. So the unique additive mapping $T:{ }_{B} M_{1} \rightarrow{ }_{B} M_{2}$ is an $\mathbb{R}$-linear mapping satisfying the conditions given in the statement, as desired.

Remark 2.2. If the second inequality in the statement of Theorem 2.1 is replaced by

$$
\left\|f\left(a^{d} x+y\right)-a^{s} f(x)-f(y)\right\| \leq \varphi(x, y),
$$

then

$$
\begin{aligned}
& \left\|f\left(a^{d} x+x\right)-a^{s} f(x)-f(x)\right\| \leq \varphi(x, x), \\
& \left\|f\left(a^{d} x+x\right)-f\left(a^{d} x\right)-f(x)\right\| \leq \varphi\left(a^{d} x, x\right) .
\end{aligned}
$$

So

$$
\left\|f\left(a^{d} x\right)-a^{s} f(x)\right\| \leq \varphi(x, x)+\varphi\left(a^{d} x, x\right),
$$

hence the result does also hold.

\section{Stability of the Pexider Functional Equation in Banach Modules}

In this section, we prove the Hyers-Ulam-Rassias stability of the Pexider functional equation in Banach modules over a unital Banach algebra.

Theorem 3.1. Let $f, g, h:{ }_{B} M_{1} \rightarrow{ }_{B} M_{2}$ be mappings for which there exists a function $\varphi:{ }_{B} M_{1} \backslash\{0\} \times{ }_{B} M_{1} \backslash\{0\} \rightarrow[0, \infty)$ such that

$$
\begin{gathered}
\widetilde{\varphi}(x, y):=\sum_{k=0}^{\infty} 3^{-k} \varphi\left(3^{k} x, 3^{k} y\right)<\infty, \\
\left\|f\left(a^{d} x+a^{d} y\right)-a^{s} g(x)-a^{s} h(y)\right\| \leq \varphi(x, y), \\
\|f(t x+t y)-t g(x)-t h(y)\| \leq \varphi(x, y)
\end{gathered}
$$

for all $a \in B_{1}$, all $t \in \mathbb{R}^{+}$, and all $x, y \in{ }_{B} M_{1} \backslash\{0\}$. Then there exists a unique $\mathbb{R}$-linear mapping $T:{ }_{B} M_{1} \rightarrow{ }_{B} M_{2}$ such that

$$
\begin{aligned}
T\left(a^{d} x\right)= & a^{s} T(x), \\
\|f(x)-f(0)-T(x)\| \leq & \frac{1}{3} \widetilde{\varphi}\left(\frac{x}{2}, \frac{-x}{2}\right)+\frac{1}{3} \widetilde{\varphi}\left(\frac{-x}{2}, \frac{x}{2}\right)+\frac{1}{3} \widetilde{\varphi}\left(\frac{x}{2}, \frac{x}{2}\right)+\frac{2}{3} \widetilde{\varphi}\left(\frac{-x}{2}, \frac{x}{2}\right) \\
& +\frac{1}{3} \widetilde{\varphi}\left(\frac{-x}{2}, \frac{3 x}{2}\right)+\frac{1}{3} \widetilde{\varphi}\left(\frac{3 x}{2}, \frac{-x}{2}\right)+\frac{1}{3} \widetilde{\varphi}\left(\frac{3 x}{2}, \frac{3 x}{2}\right)
\end{aligned}
$$

for all $a \in B_{1}$ and all $x \in{ }_{B} M_{1} \backslash\{0\}$.

Proof. Put $a=1$. By [7, Theorem 2.2], there exists a unique additive mapping $T:{ }_{B} M_{1} \rightarrow{ }_{B} M_{2}$ satisfying (iv). The mapping $T:{ }_{B} M_{1} \rightarrow{ }_{B} M_{2}$ was given by

$$
T(x)=\lim _{n \rightarrow \infty} \frac{f\left(3^{n} x\right)}{3^{n}}=\lim _{n \rightarrow \infty} \frac{g\left(3^{n} x\right)}{3^{n}}=\lim _{n \rightarrow \infty} \frac{h\left(3^{n} x\right)}{3^{n}}
$$


for all $x \in{ }_{B} M_{1}$. For each fixed $a \in B_{1}$, it follows from the second inequality of the statement and the definition of the mapping $T$ that

$$
T\left(a^{d} x\right)=\lim _{n \rightarrow \infty} \frac{f\left(3^{n} a^{d} x\right)}{3^{n}}=\lim _{n \rightarrow \infty} \frac{a^{s} g\left(3^{n} x\right)}{3^{n}}=a^{s} T(x)
$$

for all $a \in B_{1}$ and all $x \in{ }_{B} M_{1}$.

Similarly, one can obtain that

$$
T(t x)=\lim _{n \rightarrow \infty} \frac{f\left(3^{n} t x\right)}{3^{n}}=\lim _{n \rightarrow \infty} \frac{t f\left(3^{n} x\right)}{3^{n}}=t T(x)
$$

for each $t \in \mathbb{R}^{+}$.

The rest of the proof is the same as the proof of Theorem 2.1. So the unique additive mapping $T:{ }_{B} M_{1} \rightarrow{ }_{B} M_{2}$ is an $\mathbb{R}$-linear mapping satisfying the conditions given in the statement, as desired.

Corollary 3.2. Let $p<1$, and $f, g, h:{ }_{B} M_{1} \rightarrow{ }_{B} M_{2}$ mappings such that

$$
\begin{aligned}
\left\|f\left(a^{d} x+a^{d} y\right)-a^{s} g(x)-a^{s} h(y)\right\| & \leq\|x\|^{p}+\|y\|^{p}, \\
\|f(t x+t y)-t g(x)-t h(y)\| & \leq\|x\|^{p}+\|y\|^{p}
\end{aligned}
$$

for all $a \in B_{1}$, all $t \in \mathbb{R}^{+}$, and all $x, y \in{ }_{B} M_{1} \backslash\{0\}$. Then there exists a unique $\mathbb{R}$-linear mapping $T:{ }_{B} M_{1} \rightarrow{ }_{B} M_{2}$ such that

$$
\|f(x)-f(0)-T(x)\| \leq \frac{4\left(3+3^{p}\right)}{2^{p}\left(3-3^{p}\right)}\|x\|^{p}, \quad T\left(a^{d} x\right)=a^{s} T(x)
$$

for all $a \in B_{1}$ and all $x \in{ }_{B} M_{1} \backslash\{0\}$.

Proof. Define $\varphi(x, y)=\|x\|^{p}+\|y\|^{p}$, and apply Theorem 3.1.

Now we prove the Hyers-Ulam-Rassias stability of another Pexider functional equation in Banach modules over a unital Banach algebra.

Theorem 3.3. Let $f, g, h:{ }_{B} M_{1} \rightarrow{ }_{B} M_{2}$ be mappings for which there exists a function $\varphi:{ }_{B} M_{1} \backslash\{0\} \times{ }_{B} M_{1} \backslash\{0\} \rightarrow[0, \infty)$ satisfying (iii) such that

$$
\begin{aligned}
\left\|a^{s} f(x+y)-g\left(a^{d} x\right)-h\left(a^{d} y\right)\right\| & \leq \varphi(x, y), \\
\|t f(x+y)-g(t x)-h(t y)\| & \leq \varphi(x, y)
\end{aligned}
$$

for all $a \in B_{1}$, all $t \in \mathbb{R}^{+}$, and all $x, y \in{ }_{B} M_{1} \backslash\{0\}$. Then there exists a unique $\mathbb{R}$-linear mapping $T:{ }_{B} M_{1} \rightarrow{ }_{B} M_{2}$ satisfying (iv) such that

$$
a^{s} T(x)=T\left(a^{d} x\right)
$$

for all $a \in B_{1}$ all $x \in{ }_{B} M_{1} \backslash\{0\}$. 
Proof. By the same reasoning as the proof of Theorem 3.1, there exists a unique additive mapping $T:{ }_{B} M_{1} \rightarrow{ }_{B} M_{2}$ satisfying (iv). For each fixed $a \in B_{1}$, it follows from the first inequality of the statement and the definition of the mapping $T$ that

$$
a^{s} T(x)=\lim _{n \rightarrow \infty} \frac{a^{s} f\left(3^{n} x\right)}{3^{n}}=\lim _{n \rightarrow \infty} \frac{g\left(3^{n} a^{d} x\right)}{3^{n}}=T\left(a^{d} x\right)
$$

for all $a \in B_{1}$ and all $x \in{ }_{B} M_{1}$.

The rest of the proof is similar to the proof of Theorem 2.1. So the unique additive mapping $T:{ }_{B} M_{1} \rightarrow{ }_{B} M_{2}$ is an $\mathbb{R}$-linear mapping satisfying the conditions given in the statement, as desired.

Similarly, one can prove the stability of the other linear functional equations in Banach modules over a unital Banach algebra.

\section{References}

[1] J. Aczél and J. K. Chung, Integrable solutions of functional equations of a general type, Studia Sci. Math. Hungar. 17(1982), 51-67.

[2] F. Bonsall and J. Duncan, Complete Normed Algebras, Springer-Verlag, New York, Heidelberg and Berlin, 1973.

[3] Z. Gajda, On stability of additive mappings, Internat. J. Math. Math. Sci. 14(1991), 431-434.

[4] P. Găvruta, A generalization of the Hyers-Ulam-Rassias stability of approximately additive mappings, J. Math. Anal. Appl. 184(1994), 431-436.

[5] D. H. Hyers, On the stability of the linear functional equation, Pro. Nat'l. Acad. Sci. U.S.A. 27(1941), 222-224.

[6] D. H. Hyers, G. Isac and Th. M. Rassias, Stability of Functional Equations in Several Variables, Birkhäuser, Berlin, Basel and Boston, 1998.

[7] K. Jun and Y. Lee, A generalization of the Hyers-Ulam-Rassias stability of Pexider equation, J. Math. Anal. Appl. 246(2000), 627-638.

[8] K. Kovács, On the characterization of additive and multiplicative functions, Studia Sci. Math. Hungar. 18(1983), 1-11.

[9] Th. M. Rassias, On the stability of the linear mapping in Banach spaces, Proc. Amer. Math. Soc. 72(1978), 297-300.

[10] H. Schröder, $K$-theory for real $C^{*}$-algebras and applications, Pitman Research Notes in Math. Ser., Longman Sci. Tech. Essex, 290(1993).

[11] F. Skof, Proprietà locali e approssimazione di operatori, Rend. Sem. Mat. Fis. Milano 53(1983), 113-129.

[12] S. M. Ulam, Problems in Modern Mathematics, Wiley, New York, 1960.

Department of Mathematics, Chungnam National University, DaeJeon 305-764, South Korea. E-mail: cgpark@math.cnu.ac.kr 